\title{
EL CONSUMO Y LA LÍNEA DE POBREZA EN ANTIOQUIA: APROXIMACIONES MEDIANTE LA TEORÍA DEL CONSUMIDOR*
}

\author{
Juan Camilo Galvis Ciro** \\ Recibido: enero 31 de 2011 \\ Aceptado: mayo 14 de 2012
}

\section{RESUMEN}

Este documento presenta un análisis de la magnitud, composición y distribución del gasto de los hogares antioqueños en el año 2008 haciendo uso de los datos de la encuesta de calidad de vida realizada por el DANE. Se calcula el consumo de subsistencia o línea de pobreza para la región de Antioquia a través de la estimación de un sistema cuadrático de gastos. También se construye una serie de precios implícitos a partir del gasto de los hogares con el propósito de calcular elasticidades precio y gasto de la demanda. Mediante la ley de Engel se encuentra que hay mayor calidad de vida en la zona urbana de Antioquia y los resultados muestran que el gasto mínimo de subsistencia en doce diferentes grupos de bienes para la región de Antioquia es de $\$ 356,994.63$, a pesos del 2008.

\section{PALABRAS CLAVE}

Línea de pobreza, consumo, ley de Engel, pobreza, Antioquia (Colombia).

\section{CLASIFICACIÓN JEL}

\author{
D12, I32, I39
}

\section{CONTENIDO}

Introducción; 1. La teoría neoclásica del consumidor; 2. Información utilizada para estimar el sistema; 3. Magnitud y distribución del gasto; 4. La línea de pobreza para Antioquia; 5. Elasticidades precio propio, cruzada y gasto de la demanda; 6.Comentarios finales; Bibliografía; Anexos.

\footnotetext{
Este artículo de investigación es producto del proyecto titulado "Estimación de la línea de pobreza para Antioquia", adscrito al Grupo de Investigación de Microeconomía Aplicada y Teoría Económica (Clasificado en categoría D en Colciencias) del Departamento de Economía, Facultad de Ciencias Humanas y Económicas, Universidad Nacional de Colombia, Medellín, Colombia. El proyecto fue financiado por la Vicedecanatura de Extensión e Investigación en el 2011.

** Economista y Magíster en Ciencias Económicas, Facultad de Ciencias Humanas y Económicas, Universidad Nacional de Colombia, Medellín, Colombia. Miembro Grupo de Investigación de Microeconomía Aplicada y Teoría Económica, Facultad de Ciencias Humanas y Económicas, Universidad Nacional. Dirección postal: Calle 59a No. 63-20, Bloque 46, Facultad de Ciencias Humanas Económicas, Universidad Nacional de Colombia, Medellín, Colombia. Correo electrónico: jcgalvis@unal.edu.co.
} 


\title{
CONSUMPTION AND THE POVERTY LINE IN ANTIOQUIA: CONSUMER CHOICE APPROXIMATIONS
}

\section{ABSTRACT}

This paper analyses the magnitude, composition and expenditure distribution of households in Antioquia during 2008 based on the quality of life survey carried out by DANE. The subsistence consumption or poverty line for the Antioquia region is calculated with a quadratic expenditure system. A series of implicit prices is also constructed utilising household expenditures with the aim of calculating price elasticities and demand expenditures. According to Engel's law there is a better quality of life in the urban zones of Antioquia and the results show that the minimum subsistence expenditure in twelve different groups of goods for the Antioquia region is $\$ 356,994.63$ in 2008 Colombian pesos.

\section{KEY WORDS}

Poverty line, Consumption, Engel's law, Poverty, Antioquia (Colombia)

\section{JEL CLASSIFICATION}

\author{
D12, I32, 139
}

\section{CONTENT}

Introduction; 1 . Theory of neoclassic consumer choice; 2 . Information used for estimating the system; 3. Expenditure magnitude and distortion; 4. Poverty line for Antioqua; 5. Price elasticity, cross elasticity and demand expenditure; 6. Final comments; Bibliography; Attachments.

\section{CONSUMO E LINHA DE POBREZA EM ANTIOQUIA: ABORDAGENS ATRAVÉS DA TEORIA DE CONSUMIDOR}

\section{RESUMO}

Este documento apresenta uma análise da magnitude, composição e distribuição do gasto dos lares antioquenhos no ano 2008 fazendo uso dos dados da enquete de qualidade de vida realizada pelo DANE. Calcula-se o consumo de subsistência ou linha de pobreza para a região de Antioquia a través da estimação de um sistema quadrático de gastos. Também se constrói uma serie de preços implícitos a partir do gasto dos lares com o propósito de calcular elasticidades de preço e gasto da demanda. Mediante a lei de Engel encontra-se que se tem maior qualidade de vida na zona urbana de Antioquia e os resultados mostram que o gasto mínimo de subsistência em doze diferentes grupos de bens para a região de Antioquia é de $\$ 356,994.63$, a pesos do 2008 .

\section{PALAVRAS-CHAVE}

Linha de pobreza, Consumo, lei de Engel, Pobreza, Antioquia (Colômbia).

\section{CLASSIFICAÇÃO JEL}

D12, I32, I39

\section{CONTEUDO}

Introdução; 1. A teoria neoclássica do consumidor; 2. Informação utilizada para estimar o sistema; 3. Magnitude e distribuição do gasto; 4. A linha de pobreza para Antioquia; 5. Elasticidades de preço próprio, cruzada e gasto da demanda; 6. Comentarios finais; Bibliografia; Anexos. 


\section{INTRODUCCIÓN}

Es importante definir el estilo de vida compartido o aprobado en cada sociedad, para evaluar si hay un punto en la escala de distribución de recursos por debajo del cual las familias encuentran dificultades crecientes para compartir las costumbres, actividades y dietas que conforman el estilo de vida en una sociedad (Sen, 1992).

Para caracterizar dicho estilo de vida, existen desde luego muchas variables que se pueden tener en cuenta. De estas, una de las más importantes es el gasto de los hogares ya que este es el que mejor refleja las costumbres de una sociedad. De hecho, el ingreso que una persona dedica a la compra de bienes y servicios para satisfacer diversas necesidades sirve no solo como un instrumento burdo para predecir su consumo actual, sino como un indicador de su capacidad para satisfacer ciertas necesidades mínimas.

Estudiar el consumo tiene entonces potenciales aplicaciones prácticas y es por ello que gran mayoría de los estudios económicos sobre pobreza han relacionado aquella con el estándar de vida, eligiéndose para esta opción el consumo de bienes y servicios de los hogares por ser un indicador idóneo para medir el bienestar.

Para el interés de esta investigación basta recalcar que el término estándar de vida no se refiere solo a privaciones predeterminadas, sino también al hecho de no tener capacidad de llevar los hábitos de consumo imperantes en un momento de tiempo en una comunidad. Es por ello que existe una tradición económica de medir la pobreza con la variable consumo, construir líneas de pobreza a partir del costo de las necesidades estandarizadas en una comunidad, y considerar pobres a las personas cuyo ingreso o consumo no es suficiente para mantener un nivel de vida considerado mínimo (Feres y Mancero, 2001; Sen, 2000).

Es decir, del gasto de línea de pobreza de una sociedad se pueden derivar normas de comportamientos típicas. Por lo tanto, es un método basado en capacidades y sirve para definir los pobres con base en quienes no tienen capacidad de satisfacer las necesidades, dentro de las restricciones de comportamiento típicas de su sociedad (Sen, 2000). Ha sido este carácter práctico de definir la calidad de vida con los gastos en bienes y servicios, por lo que el estudio del consumo de los hogares siempre reviste interés.

Teniendo en cuenta lo anterior, se analizará a partir de las herramientas de la teoría del consumidor, el consumo y la línea de pobreza en Antioquia. Para ello, se estudiará el consumo de los hogares mediante la utilización de sistemas de ecuaciones de demanda ya que estos permiten analizar, con base en las proposiciones teóricas y la evidencia empírica, la forma como las familias realizan sus asignaciones de consumo en los diferentes grupos de bienes y servicios. 
En particular, se utiliza el sistema cuadrático de gastos para realizar una estimación de la línea de pobreza que posibilite determinar el estándar de vida existente en Antioquia. Luego se utiliza el sistema casi ideal de demanda (AIDS) para estimar elasticidades gasto y precio de la demanda. Para ello, se utilizan los datos de la encuesta de calidad de vida del 2008 realizada por el Departamento Administrativo Nacional de Estadística (DANE) concernientes a las familias de la región de Antioquia, y se construye una serie de pseudo precios implícitos mediante el uso de la metodología propuesta por Perali, Atella y Menon (2004), la cual fue aplicada hace poco para Colombia por Ballesteros (2011).

El documento está dividido en seis secciones, sin incluir esta introducción. En la primera sección se presenta una revisión de los principales aspectos de la teoría del consumidor, en especial del marco teórico en donde se desarrolla el sistema cuadrático de gastos. En la segunda, se da a conocer algunos conceptos metodológicos que se utilizan para estudiar el consumo de los hogares antioqueños tales como la clasificación de grupos de gastos a utilizar. En la tercera parte, se estudia la composición y magnitud del gasto de los hogares. En la cuarta, se estima una línea de pobreza a partir de esto gastos. En la quinta parte, se realiza la estimación de un sistema de demanda del tipo flexible con el fin de cuantificar diferentes elasticidades y, por último, vienen las conclusiones.

\section{LA TEORÍA NEOCLÁSICA DEL CONSUMIDOR}

Para analizar el consumo de los hogares, se especificarán sistemas completos de ecuaciones de demanda los cuales tienen su sustento teórico en la teoría neoclásica del consumidor.

Esta teoría económica parte de la idea central de la racionalidad del agente, en el sentido en que este busca su máxima satisfacción posible en su conjunto factible de bienes. Se supone entonces que los agentes tienen un comportamiento optimizador.

El conjunto costeable es el llamado conjunto de restricción presupuestario que por lo general asume las características de conjunto presupuestario walrasiano. La racionalidad implica que el agente es capaz de elegir canastas, es coherente en sus decisiones y que, además, la elección busca satisfacer las preferencias al máximo. Bajo ciertas condiciones adicionales, el problema de elección del agente puede plantearse como la maximización de una función llamada de utilidad sujeto a un presupuesto, y de este problema salen como resultado las demandas de bienes y servicios del consumidor (Mas-Colell, Whiton y Green, 1995).

Desde la perspectiva del análisis empírico de la demanda, la teoría del consumidor sirve para proveer unas ricas fuentes de formas funcionales especificadas 
en sistemas de demanda que pueden servir como hipótesis de estimación para verificar elasticidades, simetrías del efecto sustitución cruzado, negatividad del efecto sustitución, agotamiento del gasto, entre otras.

Las restricciones que la teoría implica para una conducta racional pueden ser impuestas a los sistemas y verificadas luego con los datos. Algunos sistemas, como el sistema lineal de gastos (Stone, 1954) el sistema cuadrático de gastos (Howe, Pollak y Wales, 1979), el sistema de Rotterdam y el sistema casi ideal de demanda (Deaton y Muellbauer, 1980), por ejemplo, hacen uso de funciones de utilidad directa, indirecta o funciones de gasto para especificar formas específicas de las funciones de demanda con el objetivo de verificar algunas proposiciones teóricas.

\subsection{El sistema cuadrático de gastos}

Varios trabajos se han desarrollado con el fin de especificar formas funcionales de sistemas de demanda que puedan ser usados para trabajar con los datos disponibles de los ingresos y gastos de las familias. En este documento, se estimará en una primera parte el llamado sistema cuadrático de gastos para la región de Antioquia.

El sistema cuadrático de gastos fue postulado por Nicholson (1949) quien estimó funciones cuadráticas de las curvas de Engel con ecuaciones adhoc. Más tarde, sería Howe (1974) quien demostrara que las funciones de ingreso-consumo cuadráticas podían ser derivadas de un proceso de maximización de la utilidad, y que estas funciones en su forma reducida eran idénticas a un sistema de gastos. Pocos años después, serían Howe, Pollak y Wales (1979) quienes generalizarían el sistema cuadrático de gastos.

El sistema cuadrático parte de la siguiente función de utilidad indirecta:

$$
\psi(p, m)=\frac{-g(p)}{m-b(p)}+\frac{g(p)}{f(p)}
$$

Donde $m$ es la renta y $g(p), b(p), f(p)$ son funciones homogéneas de grado uno en precios. Si se utiliza la identidad de Roy en la función anterior, es posible encontrar la cantidad demandada del bien $i$. La identidad es la siguiente:

$$
x_{i}(p, m)=-\frac{\frac{\partial \psi(p, m)}{\partial p_{i}}}{\frac{\partial \psi(p, m)}{\partial m}}
$$


Por lo tanto, se tiene:

$$
\begin{aligned}
& x_{i}(p, m)=-\frac{\left[\frac{-g^{\prime}(p)(m-b(p))-g(p) b^{\prime}(p)}{(m-b(p))^{2}}+\frac{g^{\prime}(p) f(p)-g(p) f^{\prime}(p)}{(f(p))^{2}}\right]}{\frac{g(p)}{(m-b(p))^{2}}} \\
& x_{i}(p, m)=\frac{\frac{g^{\prime}(p)(m-b(p))+g(p) b^{\prime}(p)}{(m-b(p))^{2}}-\frac{1}{f(p)}\left[g^{\prime}(p)-\frac{g(p) f^{\prime}(p)}{f(p)}\right]}{\frac{g(p)}{(m-b(p))^{2}}} \\
& x_{i}(p, m)=\frac{g^{\prime}(p)(m-b(p))+g(p) b^{\prime}(p)}{g(p)}-\frac{(m-b(p))^{2}}{g(p) f(p)}\left(g^{\prime}(p)-\frac{g(p)}{f(p)} g^{\prime}(p)\right) \\
& x_{i}(p, m)=b^{\prime}(p)+\frac{g^{\prime}(p)}{g(p)}(m-b(p))-\left(\frac{g^{\prime}(p)-g(p) \frac{f^{\prime}(p)}{f(p)}}{f(p) g(p)}\right)(m-b(p))^{2}
\end{aligned}
$$

Se comprueba que $\chi_{i}(p, m)$ es homogénea de grado cero en $(p, m)$. Esta ecuación es la demanda del bien $i$ en el sistema cuadrático de gastos. Se puede definir, con base en Samuelson (1947), a $b(p)=\sum p_{i} \gamma_{i}$ y $b^{\prime}(p)=\gamma_{i}$ como el gasto mínimo de subsistencia en el bien $i$.

En este sentido, se demuestra que tanto el sistema cuadrático de gastos como el llamado sistema lineal de gastos, se pueden utilizar para construir umbrales de pobreza a partir de la estimación de los consumos de subsistencia para un determinado grupo de hogares.

Se puede comprobar también bajo qué condiciones se cumple la condición de agotamiento del gasto. El sistema de demanda es:

$x_{i}(p, m)=b^{\prime}(p)+\frac{g^{\prime}(p)}{g(p)}(m-b(p))-\left(\frac{g^{\prime}(p) g(p) \frac{f^{\prime}(p)}{f(p)}}{f(p) g(p)}\right)(m-b(p))^{2}$ 
Y si simplifica éste se tiene:

$x_{i}(p, m)=\gamma_{i}+\beta_{i}\left(m-\sum \gamma_{j}\right)+\delta_{i}\left(m-\sum \gamma_{j}\right)^{2}$

Donde $b^{\prime}(p)=\gamma_{i}, \frac{g^{\prime}(p)}{g(p)}=\beta_{i}$ y $\left(\frac{g^{\prime}(p) g(p) \frac{f^{\prime}(p)}{f(p)}}{f(p) g(p)}\right)=\delta_{i}$

Multiplicando a ambos lados por $p_{i}$ se llega a:

$$
p_{i} x_{i}(p, m)=p_{i} \gamma_{i}+\beta_{i}\left(m-\sum p_{j} \gamma_{j}\right)+\delta_{i}\left(m-\sum p_{j} \gamma_{j}\right)^{2}
$$

La condición de agotamiento del gasto, definida como:

$\sum p_{i} x_{i}(p, m)=m$

Se cumple en el sistema cuando:

$$
\sum p_{i} x_{i}(p, m)=\sum p_{i} \gamma_{i}+\sum \beta_{i}\left(m-\sum p_{j} \gamma_{j}\right)+\sum \delta_{i}\left(m-\sum p_{j} \gamma_{j}\right)^{2}=m
$$

Es decir, sí y sólo sí:

$$
\sum \beta_{i}=1 \text { y } \sum \delta_{i}=0 \text { ya que en éste caso se tiene: }
$$

$$
\sum p_{i} x_{i}(p, m)=\sum p_{i} \gamma_{i}+m-\sum p_{i} \gamma_{i}=m
$$

Además, el sistema cumple también con lo que se conoce como las condiciones de integrabilidad.

\subsection{Sistema reducido}

Una vez verificadas las condiciones que impone la teoría del consumidor en el sistema, se intenta a continuación reducirlo en una ecuación que sea posible estimar. Sea:

$$
p_{i} x_{i}(p, m)=p_{i} \gamma_{i}+\beta_{i}\left(m-\sum p_{j} \gamma_{j}\right)+\delta_{i}\left(m-\sum p_{j} \gamma_{j}\right)^{2}
$$

Si se supone $b(p)=\sum p_{i} \gamma_{i}=b$ se tiene:

$$
q_{i}=b+\beta_{i}\left(m-\sum p_{j} \gamma_{j}\right)+\delta_{i}\left(m-\sum p_{j} \gamma_{j}\right)^{2}
$$


Donde $q_{i}=p_{i} \chi_{i}(p, m)$ es el gasto en el bien $i$.

Se puede considerar, al igual que en el sistema lineal de gastos, a 6 como el consumo de subsistencia, y $\left(m-\sum p_{j} \gamma_{j}\right)$ puede ser considerado como el monto del ingreso que está por encima del ingreso de subsistencia. Por último, $\left(m-\sum p_{j} \gamma_{j}\right)^{2}$ intenta captar no linealidades en la curva de ingreso-consumo. Si $\beta_{i}$ es positivo se considera al bien $i$ como normal y si es negativo sería inferior.

Si se realiza el producto de la parte derecha del sistema, se tiene:

$$
\begin{aligned}
& q_{i}=b+\beta_{i} m-\beta_{i} \sum b_{k}+\delta_{i} m^{2}-2 \delta_{i} m \sum b_{k}+\delta_{i}\left(\sum b_{k}\right)^{2} \\
& q_{i}=b-\beta_{i} \sum b_{k}+\delta_{i}\left(\sum b_{k}\right)^{2}+\left(\beta_{i}-2 \delta_{i} \sum b_{k}\right) m+\delta_{i} m^{2}
\end{aligned}
$$

En forma reducida la ecuación a estimar sería:

$$
q_{i}=\theta_{i 1}+\theta_{i 2} m+\delta_{i} m^{2}
$$

Donde:

$$
\begin{aligned}
& \theta_{i 1}=b-\beta_{i} \sum b_{k}+\delta_{i}\left(\sum b_{k}\right)^{2} \\
& \theta_{i 2}=\left(\beta_{i}-2 \delta_{i} \sum b_{k}\right)
\end{aligned}
$$

El término al cuadrado, que distingue al sistema cuadrático de gastos del sistema lineal de gastos, permite captar la no linealidad de la función ingreso-consumo lo que hace que el sistema tome en cuenta las variaciones en la participación del gasto de cada bien a medida que el nivel de ingreso cambia'. Por ejemplo, si en las estimaciones el parámetro que acompaña el término al cuadrado es negativo, se tendría que la participación de este bien sobre el gasto total desciende a medida que varía el ingreso (Howe, 1974).

Es decir, el sistema cuadrático de gastos tiene una ventaja respecto al sistema lineal de gastos ya que en este las funciones de ingresos consumo necesitan ser lineales, lo que significa que el porcentaje de un peso adicional del ingreso es distribuido de manera igual para todos los hogares de cada grupo, sin importar el nivel de ingreso de cada hogar. No obstante, a menudo las curvas de ingreso consumo no son lineales.

Es posible afirmar, además, que el sistema cuadrático intenta corregir los problemas de heteroscedasticidad que presenta la estimación del sistema lineal de

\footnotetext{
Muñoz, Ramírez y Zambrano (2005) estiman curvas de Engel y encuentran, tanto en estimaciones paramétricas como no paramétricas, que las curvas para diferentes bienes en la economía colombiana son no lineales.
} 
gastos. Desde que este no tiene en cuenta los términos al cuadrado que distinguen al sistema cuadrático, si se encuentra que las estimaciones de estos términos son significativas, existirán indicios de algunos problemas econométricos del sistema lineal de gastos. Es decir, el sistema cuadrático es un buen intento de tener un punto medio entre buen comportamiento estadístico y buen comportamiento teórico.

\subsection{Problemas de especificación}

Especificadas las demandas del sistema cuadrático de gastos en una ecuación reducida, lo ideal sería hacer la estimación. El problema es que el sistema presenta problemas de identificación que se deben resolver antes de estimarlo.

El sistema en forma reducida es:

$$
q_{i}=\theta_{i 1}+\theta_{i 2} m+\delta_{i} m^{2}
$$

Se tiene entonces que hay $n 6$ y otros $n \beta_{i}$, es decir, $2 n$ incógnitas con $2 n$ ecuaciones, por lo que es necesario definir una ecuación adicional para especificar bien el sistema a la hora de la estimación. Además, $<<$ sem-6-32.wmf $>>$ son ecuaciones linealmente dependientes. Es decir, hay una ecuación que es dependiente de las otras por lo que es necesario incluir una ecuación adicional, que en este caso será la ecuación para el ahorro.

Sea:

$$
q_{s}=b_{s}+\beta_{s}\left(m-\sum b_{k}\right)+\delta_{s}\left(m-\sum b_{k}\right)^{2}
$$

Donde $q_{2}$ es el gasto en bienes durables y será nuestra ecuación para el ahorro. Para ello se descompone la ecuación anterior, la cual queda:

$$
q_{s}=b_{s}-\beta_{s} \sum b_{k}+\delta_{s}\left(\sum b_{k}\right)^{2}+\left(\beta_{s}-2 \delta_{s} \sum b_{k}\right) m+\delta_{s} m^{2}
$$

Se va a suponer que $b_{\varsigma}$ igual a cero y se supone así que la propensión marginal a ahorrar es igual a cero para especificar bien el sistema. Con ello, se tiene lo siguiente:

$$
q_{s}=-\beta_{s} \sum b_{k}+\delta_{s}\left(\sum b_{k}\right)^{2}+\left(\beta_{s}-2 \delta_{s} \sum b_{k}\right) m+\delta_{s} m^{2}
$$

En forma reducida el sistema sería:

$$
q_{s}=-\theta_{s 1}+\theta_{s 2} m+\delta_{s} m^{2}
$$

Donde:

$$
\theta_{s 1}=-\beta_{s} \sum b_{k}+\delta_{s}\left(\sum b_{k}\right)^{2}
$$




$$
\theta_{s 2}=\left(\beta_{s}-2 \delta_{s} \sum b_{k}\right)
$$

Se debe tener en cuenta, que para poder encontrar los parámetros del sistema es necesario encontrar a $\beta_{s}$. Esta es igual a

$$
\beta_{s}=\theta_{s 2}+2 \delta_{s} \sum b_{k}
$$

Si se lleva esta ecuación a $\theta_{s 1}$ se tiene:

$$
\begin{aligned}
& \theta_{s 1}=-\left(\theta_{s 2}+2 \delta_{s} \sum b_{k}\right) \sum b_{k}+\delta_{s}\left(\sum b_{k}\right)^{2} \\
& \theta_{s 1}=-\theta_{s 2} \sum b_{k}-\delta_{s}\left(\sum b_{k}\right)^{2}
\end{aligned}
$$

Por lo tanto, se llega a

$$
\delta_{s}\left(\sum b_{k}\right)^{2}+\theta_{s 2} \sum b_{k}-\theta_{s 1}=0
$$

Ésta última ecuación es de la forma $a x^{2}+b c+c$ y permite identificar a los $b_{k}$ restantes del sistema. De esta manera, si se soluciona el sistema para $\sum b_{k}$ se llega a lo siguiente:

$$
\sum b_{k}=\left(-\theta_{s 2} \pm \sqrt{\theta_{s 2}^{2}-4 \delta_{s}\left(-\theta_{s 1}\right)}\right) / 2 \delta_{s}
$$

Esta ecuación es la que permite identificar los $\sigma_{i}$ del sistema y, por lo general, es aproximada mediante la estimación del sistema con bienes durables, ya que se puede suponer de manera ad hoc que el gasto mínimo de subsistencia en bienes durables es cero.

El sistema a estimar en su versión estocástica sería entonces:

$$
q_{i h}=\theta_{i 1}+\theta_{i 2} m_{h}+\delta_{i} m_{h}^{2}+e_{i h}
$$

Con $q_{i n}$ correspondiente al gasto en el bien $i$ del h-ésimo hogar, $m_{h}$ es la renta del h-ésimo hogar y $e_{i \hbar}$ son la perturbaciones que provienen de un distribución normal n-variada con la matriz de varianza-covarianza con supuestos clásicos.

\section{INFORMACIÓN UTILIZADA PARA ESTIMAR EL SISTEMA}

Para el desarrollo de esta investigación, se utilizó la información de la Encuesta de Calidad de Vida (ECV) del año 2008 realizada por el DANE, en lo que respecta al rubro de gastos de las familias. Esta encuesta fue realizada entre agosto y octubre de 2008 a 13,611 personas de Colombia que contabilizan, mediante el factor de 
expansión de la encuesta, 43,836,556 habitantes. La encuesta es representativa para diversas regiones, entre ellas, Antioquia, donde se centra esta investigación. En esta región, la encuesta fue hecha a 1839 personas que contabilizan 5,903,053 habitantes.

Por lo general, la investigación aplicada en Colombia sobre el consumo de los hogares ha clasificado los gastos en bienes y servicios en dos grupos: gastos corrientes y gastos no corrientes. El primero está compuesto por el gasto en once grupos, y el gasto no corriente está compuesto por dos grupos. Se tienen así trece grupos de gastos.

A pesar de esta tradición en el trabajo empírico, esta investigación se debe apartar de ella debido a que se va a construir una serie de precios mediante el método de Lewbel (1989) y Perali, Atella y Menon (2004) donde es necesario, por la exigencia en información del método, agrupar los bienes de tal forma que se evite la mayor cantidad de gastos cero posibles. Esta forma de proceder, no obstante, va acorde con el único trabajo empírico del que se tiene registro para Colombia, (Ballesteros, 2011), en el que se haya aplicado un método de construcción de precios implícitos.

Por esta razón, en esta investigación se agruparon los gastos corrientes en seis grupos y el gasto no corriente en un grupo para tener un total de siete grupos de gastos. De esta forma, cuando se estime el gasto mínimo de subsistencia y luego las elasticidades, se estimarán aquellas para los grupos de gastos que entran a formar parte de la línea de pobreza. Los grupos se describen a continuación.

\subsection{Gastos corrientes}

Alimentos. Se encuentra compuesto por el gasto en arepas, lácteos, huevos, carnes, pollo, pescada, jamón, papa, arroz, leguminosas, plátano, hortalizas, frutas, grasas, azúcar, sal, panela, café, confitería, enlatados, galletas, gaseosas, alimentos fuera del hogar.

Servicios de la vivienda o vivienda. Se encuentra compuesto por el gasto en arriendos e impuesto predial, gas natural, alcantarillado, recolección de basuras, acueducto, combustibles, telefonía fija y gasto en electricidad.

Salud. Se encuentra compuesto por el gasto en hospitalización, servicios de hospitalización, pago de la EPS, gasto en remedios, algodón, medicina prepagada, atención médica, servicios de odontología, vacunas y gasto en exámenes.

Transporte y comunicaciones. Se encuentra compuesto por gasto en transporte público y transportes indirectos, parqueadero, celular, gasolina de vehículo particular, mantenimiento de vehículo y gasto en correo. 
Educación, recreación y cultura. Está compuesto por el gasto en matrículas, uniformes, útiles escolares, libros, prensiones, transportes escolar, periódicos, recreación, suscripción a televisión, películas, hoteles, gasto en mascotas y arte.

Bebida, tabaco y servicios personales. Está compuesto por el gasto en bebidas alcohólicas, tabaco, fósforos, lustrabotas, apuestas, aseo personal, servicios lavandería, servicios de peluquería y gastos en joyas.

\subsection{Gastos no corrientes}

Bienes durables, vestido y otros pagos. Está compuesta por el gasto en muebles, reparación de vivienda, electrodomésticos, compra de vehículos, gasto en bienes raíces, juegos electrónicos, computadoras, impresoras, compras de celular, gastos en amortización, tarjetas de crédito, seguros, gasto en ropa, zapatos, telas, medias veladas, reparación de calzado y vestuario.

\section{MAGNITUD Y DISTRIBUCIÓN DEL GASTO}

Una vez definida la información que se utiliza y la clasificación de los grupos de gastos, se comienza entonces a analizar la magnitud y distribución de gastos en Antioquia. Se muestra en un primer cuadro el tamaño del gasto, calculándose para ello la magnitud de gasto total.

Cuadro 1. Gasto total para la región y por zonas para Antioquia, 2008

\begin{tabular}{|l|c|c|c|}
\hline \multicolumn{1}{|c|}{ Región - zona } & $\begin{array}{c}\text { Gasto total (miles de } \\
\text { pesos) }\end{array}$ & Hogares & Personas \\
\hline Antioquia & $2,543,203,300$ & $1,606,775$ & $5,903,053$ \\
\hline Urbano & $2,305,814,100$ & $1,251,126$ & $4,539,409$ \\
\hline Rural & $237,389,192$ & 355,649 & $1,363,644$ \\
\hline
\end{tabular}

Fuente: elaboración propia con información de la ECV, DANE (2008).

Según se observa en el cuadro 1, la región urbana contribuye con un 90\% del gasto total de la región. Esto da algunos indicios de la desigualdad entre las dos zonas, aunque es necesario tener en cuenta que la mayor parte de la población en Antioquia está ubicada en la zona urbana (76\%). Se procede ahora a analizar el gasto por deciles para tener más detalles de la distribución del gasto.

\subsection{Gasto por deciles}

Se presenta a continuación el gasto per cápita discriminado por deciles. 
El consumo y la línea de pobreza en Antioquia: aproximaciones mediante la teoría del consumidor

Cuadro 2. Gasto per cápita por deciles para Antioquia, 2008

\begin{tabular}{|l|c|}
\hline \multicolumn{1}{|c|}{ Decil } & Gasto per cápita total (en miles de pesos) \\
\hline Primer decil & $5,671,866$ \\
\hline Segundo decil & $11,963,384$ \\
\hline Tercer decil & $18,150,178$ \\
\hline Cuarto decil & $24,171,850$ \\
\hline Quinto decil & $31,911,467$ \\
\hline Sexto decil & $38,054,098$ \\
\hline Séptimo decil & $57,081,618$ \\
\hline Octavo decil & $82,578,274$ \\
\hline Noveno decil & $132,637,123$ \\
\hline Decimo decil & $363,341,301$ \\
\hline Total & $765,561,159$ \\
\hline
\end{tabular}

Fuente: elaboración propia con información de la ECV, DANE (2008).

Según el cuadro 2, hay grandes brechas entre el primer y último decil del gasto. El gasto per cápita del decil diez es 64 veces el gasto per cápita total del decil uno. Se observa en el cuadro 2 cómo el gasto per cápita total está concentrado en un $83 \%$ en los deciles séptimo, octavo, noveno y décimo. Se procede ahora a realizar algunas estadísticas del gasto per cápita promedio.

Cuadro 3. Gasto per cápita promedio por zonas para Antioquia, 2008

\begin{tabular}{|l|c|}
\hline \multicolumn{1}{|c|}{ Región - zona } & Gasto per cápita promedio (en pesos) \\
\hline Antioquia & $476,458.2$ \\
\hline Urbano & $551,013.7$ \\
\hline Rural & $214,181.9$ \\
\hline
\end{tabular}

Fuente: Elaboración propia con información de la ECV, DANE (2008).

Según el cuadro 3, el gasto per cápita promedio en la zona urbana es 2.5 veces más que en la zona rural. Esto hace que el promedio del gasto per cápita en Antioquia (como un todo) se ubique entre los gastos per cápita promedio en las dos regiones, pero más hacia los patrones de la región urbana ya que el gasto per cápita en la región como un todo (Antioquia) está un 15\% por debajo del gasto per cápita en la zona urbana, pero 2.2 veces por encima del gasto per cápita en la zona rural. Se analiza ahora la estructura por deciles. 
Cuadro 4. Gasto per cápita promedio por deciles para Antioquia, 2008

\begin{tabular}{|l|c|}
\hline \multicolumn{1}{|c|}{ Decil } & $\begin{array}{c}\text { Gasto per cápita promedio } \\
\text { (en pesos) }\end{array}$ \\
\hline Primer decil & 54,783 \\
\hline Segundo decil & $99,861.31$ \\
\hline Tercer decil & $140,529.11$ \\
\hline Cuarto decil & $178,040.52$ \\
\hline Quinto decil & $216,354.8$ \\
\hline Sexto decil & $264,308.62$ \\
\hline Séptimo decil & $325,472.08$ \\
\hline Octavo decil & $431,522.35$ \\
\hline Noveno decil & $644,962.21$ \\
\hline Decimo decil & $1,426,826.24$ \\
\hline
\end{tabular}

Fuente: elaboración propia con información de la ECV, DANE (2008).

Cuando se divide el gasto per cápita promedio por deciles, aparecen las grandes diferencias del gasto por persona entre los deciles bajos y los altos. En el caso del decil diez, el gasto por persona allí es veintiséis veces más alto que el decil uno, catorce veces más alto que el decil dos, diez veces más alto que el decil tres, ocho veces más alto que el decil cuatro y seis veces más alto que el decil cinco. Esto se suma así a la evidencia de la alta concentración del gasto para la región.

\subsection{Composición del gasto}

Se analiza ahora la composición del gasto de los hogares con base en los grupos de gastos que se formaron. Haciendo uso de la llamada Ley de Engel, es de esperarse que cuando aumento el gasto disminuya la proporción del gasto en alimentos como porcentaje del gasto total, por el hecho de que estos son bienes necesarios. Por tanto, a mayor proporción del gasto en alimentos menor calidad de vida.

Se presenta entonces el promedio del gasto en los diferentes grupos.

Cuadro 5. Promedio del gasto en los diferentes grupos para Antioquia, 2008

\begin{tabular}{|l|c|c|}
\hline \multicolumn{1}{|c|}{ Gasto } & Número de observaciones & Media (en pesos) \\
\hline Alimentos & 1839 & $488,508.25$ \\
\hline Vivienda & 1839 & $408,164.71$ \\
\hline Salud & 1839 & $128,500.13$ \\
\hline
\end{tabular}


El consumo y la línea de pobreza en Antioquia: aproximaciones mediante la teoría del consumidor

\begin{tabular}{|l|c|c|}
\hline \multicolumn{1}{|c|}{ Gasto } & Número de observaciones & Media (en pesos) \\
\hline Transporte & 1839 & $157,130.03$ \\
\hline Educación y cultura & 1839 & $97,750.4$ \\
\hline Bebidas y servicios personales & 1839 & $74,479.5$ \\
\hline Bienes durables y vestido & 1839 & 227,987 \\
\hline
\end{tabular}

Fuente: Elaboración propia con información de la ECV, DANE (2008).

Según se observa en el cuadro 5 , el gasto promedio más alto se ubica en alimentos, seguido de vivienda y el gasto en bienes durables y vestidos. El menor gasto es en el grupo de bebidas, tabaco y servicios personales.

La participación del gasto en cada grupo va muy acorde con la media del gasto presentada antes. En el cuadro 6 se presenta la participación del gasto en cada grupo de bienes y servicios como porcentaje del gasto total.

Cuadro 6. Proporción de cada grupo de gastos en el gasto total para Antioquia, 2008

\begin{tabular}{|l|c|}
\hline \multicolumn{1}{|c|}{ Gasto } & Proporción en el gasto total \\
\hline Alimentos & 0.3086 \\
\hline Vivienda & 0.2579 \\
\hline Salud & 0.0812 \\
\hline Transporte & 0.0992 \\
\hline Educación y cultura & 0.0617 \\
\hline Bebidas y servicios personales & 0.0471 \\
\hline Bienes durables y vestido & 0.144 \\
\hline Suma & $100 \%$ \\
\hline
\end{tabular}

Fuente: Elaboración propia con información de la ECV, DANE (2008).

Los resultados muestran que entre alimentos, vivienda y bienes durables se va el $70 \%$ del gasto total de los hogares. El gasto en transporte, salud, educación y bebidas representan por su parte menos del $10 \%$ del gasto total cada uno.

Es necesario recordar que un método usado, aún hoy en día, para medir o aproximar la tasa de pobreza consiste en utilizar la proporción del gasto en alimentos de las hogares (Altimir, 1979). En este caso, debido a que la proporción es de $30.86 \%$, se tendría que este sería el porcentaje de hogares pobres en Antioquia. Cuando se analiza la composición del gasto discriminado por regiones (urbana y rural), los resultados anteriores cambian en un alto grado. 
Cuadro 7. Proporción de cada grupo de gastos en la zona urbana y rural de Antioquia, 2008

\begin{tabular}{|l|c|c|}
\hline \multicolumn{1}{|c|}{ Gasto } & Zona urbana & Zona rural \\
\hline Alimentos & 0.2907 & 0.4831 \\
\hline Vivienda & 0.2618 & 0.2194 \\
\hline Salud & 0.0827 & 0.0657 \\
\hline Transporte & 0.1011 & 0.081 \\
\hline Educación y cultura & 0.0642 & 0.0374 \\
\hline Bebidas y servicios personales & 0.0455 & 0.0618 \\
\hline Bienes durables y vestido & 0.1536 & 0.0511 \\
\hline Suma & $100 \%$ & $100 \%$ \\
\hline
\end{tabular}

Fuente: Elaboración propia con información de la ECV, DANE (2008).

Según se observa en el cuadro 7, mientras en la zona urbana los alimentos participan con el 29\% del gasto, en la zona rural lo hacen con el $48 \%$. Esto da indicios de que en la zona rural hay menor calidad de vida ya que el gasto se agota en un solo grupo de bienes y los demás grupos, al bajar su participación en el gasto total, hacen que se cubran menos necesidades. Tal como se apuntaba más arriba, una aproximación a la tasa de pobreza sería así de 29.07\% de hogares pobres en la zona urbana y $48.31 \%$ de hogares pobres en la zona rural.

Para analizar algún grado de diferencia en la calidad de vida entre zonas, se puede resaltar el hecho de que, por ejemplo, el gasto en bienes durables y vestidos participa con un $15 \%$ del gasto total en la zona urbana mientras en la zona rural lo hace con el 5\% del gasto total. En el caso de educación, cultura y recreación la participación en la zona urbana es del 6\% mientras en la zona rural es del 3\%.

En síntesis, los hogares en la zona urbana tienen acceso a una mayor variedad de bienes y servicios para satisfacer una mayor cantidad de necesidades respecto al sector rural, lo cual se resume en una menor calidad de vida en dicha zona.

\section{LA LÍNEA DE POBREZA PARA ANTIOQUIA}

En la sección pasada se realizó una breve aproximación a la pobreza y desigualdad entre los hogares antioqueños mediante los gastos que realizan aquellos. En esta sección se presenta ahora una estimación de los gastos mínimos necesarios.

La línea de pobreza consiste en definir el ingreso necesario para que una persona, o una familia logren cubrir con sus gastos un adecuado acceso a bienes 
y servicios que les permita llevar el nivel de vida estándar, el cual se supone está reflejado en los gastos que hacen las familias (Muñoz, 1990).

La estimación de líneas de pobreza enfrenta múltiples problemas y obstáculos ya que no es fácil definir el ingreso mínimo que un hogar determinado requiere para su subsistencia. Además de ello, el conjunto de medios necesarios para la subsistencia cambia con el tiempo y lugar, sumado al hecho de que las necesidades son subjetivas.

Se han desarrollado múltiples soluciones y modelos para resolver estos problemas, aunque ninguna es perfecta y cada una privilegia uno que otro aspecto según lo que se defina como necesidad (Muñoz, 1990). En esta investigación se utilizará la teoría del consumidor para definir la pobreza con base en el gasto de los hogares y se hace un enfoque directo al considerarse pobre a aquellos que no satisfacen una o varias necesidades básicas, relacionándose así el bienestar con el consumo efectivamente realizado.

La canasta básica en este caso estará compuesta por los diversos bienes y servicios en que se ha agrupado el gasto de los hogares, y la línea de pobreza es el gasto necesario para adquirir esa canasta básica (Feres y Mancero, 2001). Esta línea de pobreza será aproximada mediante la estimación de un sistema de ecuaciones de demanda que permita entender los patrones de gasto de los hogares, para luego descomponer dicho patrón y obtener el valor del gasto mínimo de subsistencia.

\subsection{Estimación del sistema cuadrático de gastos}

Tal como se presentó al inicio del artículo, existen diversos sistemas de ecuaciones de demanda que permiten aproximar el gasto mínimo de subsistencia. Entre ellos los principales son el sistema lineal de gastos y el sistema cuadrático de gastos.

Se utilizará el sistema cuadrático de gastos por las ventajas económicas y estadísticas que este tiene sobre el sistema lineal de gastos². Este sistema permite capturar los cambios de curvaturas que tienen, por ejemplo, las curvas de Engel a medida que el ingreso cambia, lo cual no es posible bajo el sistema lineal de gastos. Se trae, por tanto, nuevamente el sistema cuadrático de gastos en su forma reducida y estocástica, ecuación [32]:

$$
q_{i}=\theta_{i 1}+\theta_{i 2} m+\delta_{i} m^{2}+e_{i}
$$

Con los sistemas de gastos como el lineal y el cuadrático, también se pueden estimar elasticidades. No obstante, como anotan Deaton y Muellbauer (1983) estos sistemas no son flexibles y estiman elasticidades entre cierto rango. Por ello, para el cálculo de elasticidades se decidió emprender la tarea de estimar un sistema de formas flexibles, lo que implica construir una serie de precios implícitos como se muestra más adelante. 
siendo

$$
\begin{aligned}
& \theta_{i 1}=-\beta_{s} \sum b_{k}+\delta_{s}\left(\sum b_{k}\right)^{2} \\
& \theta_{i 2}=\left(\beta_{s}-2 \delta_{s} \sum b_{k}\right)
\end{aligned}
$$

$E\left(e_{i}\right)=0, E\left(e_{i}, e_{j}\right)=0, V\left(e_{i}\right)=\Omega$, es decir, $e_{i}$ está distribuido como una normal n-variada.

La estimación se hace por mínimos cuadrados ordinarios y como Lluch y Williams (1975, p. 327) y Wooldridge (2002, p. 150) anotan, esto es equivalente a estimar el modelo por regresiones aparentemente no relacionadas (SUR). Las estimaciones se hacen en el programa SAS y los resultados se presentan a continuación.

Como se anotó antes, es necesario incluir en la estimación el gasto en bienes durables (MODEL7 del cuadro 8) para poder especificar el sistema. Después de esto se procede a calcular el gama del ahorro. En el cuadro 11 se tiene el siguiente resultado:

$$
\sum b_{k}=348,297.55
$$

Una vez encontrado a $\sum b_{k}$ se calculan los gama $i$ restantes del sistema mediante la ecuación:

$$
b_{i}=\theta_{i 1}+\beta_{i} \sum b_{k}-\delta_{i}\left(\sum b_{k}\right)^{2}
$$

En el cuadro 8 se observa por ejemplo que los $b_{i}$ para cada grupo de gastos son (cifras en pesos):

$b_{1}=\$ 188,903.7$ consumo de subsistencia para alimentos.

$b_{2}=\$ 111,558.9$ consumo de subsistencia para servicios de vivienda.

$b_{3}=\$ 4140.11$ consumo de subsistencia para salud

$b_{4}=\$ 11,563.4$ consumo de subsistencia para transporte

$b_{5}=\$ 18,378.5$ consumo de subsistencia para educación, cultura y recreación

$b_{6}=\$ 22,449.8$ consumo de subsistencia para bebidas, tabaco y servicios personales. 
El consumo y la línea de pobreza en Antioquia: aproximaciones mediante la teoría del consumidor

Cuadro 7. Estimación del SCG para Antioquia, 2008

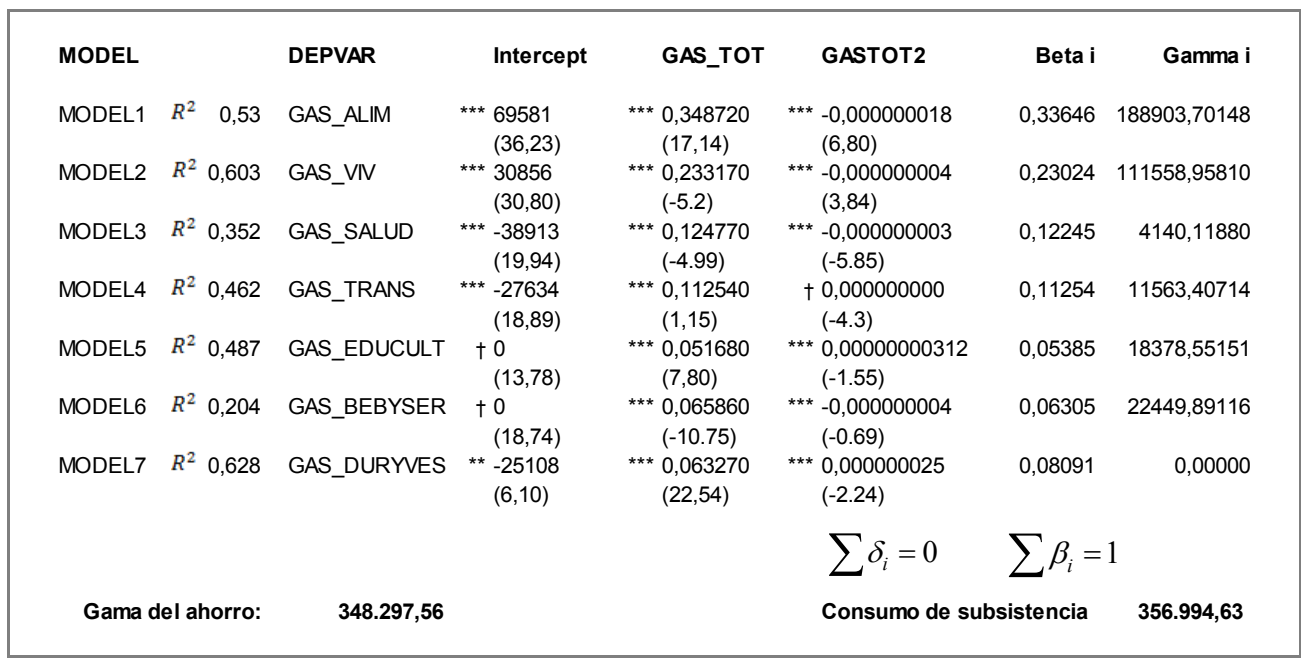

Notas: ${ }^{* * *}$ Significativo al $1 \%, * *$ Significativo al $5 \%$, † No Significativo.

Entre paréntesis están los valores t de los parámetros. Un valor t mayor a $|1.9|$ indica que el parámetro es significativo.

Fuente: Elaboración propia con información de la ECV, DANE (2008).

Por construcción $b_{7}$ es igual a cero ya que es la ecuación que se ha utilizado para identificar a las demás. Se observa entonces que el sistema arroja unos resultados aceptables. El mayor gasto de subsistencia, como era de esperarse, se hace en alimentos seguido de vivienda. Es posible que gastos en grupos de bienes como salud, transporte y educación, al estar subsidiados en algunos casos, estén subvaluados.

Es posible interpretar a los diferentes $b_{i}$ como el gasto de subsistencia en el bien i. Para el caso de alimentos, que reviste importancia para los análisis de pobreza, el sistema arroja que el gasto mínimo de subsistencia en alimentos para la región de Antioquia es igual a $\$ 188,903.7$. La suma de todos los gastos de subsistencia da un resultado de $\$ 356,994.63$ necesarios para el gasto en los diferentes bienes al mes.

Tal como se ha dicho antes, una de las formas convencionales de medir el nivel de pobreza de un país es utilizar el nivel de ingresos de las familias y compararlos frente a un umbral, llamado valor de línea de pobreza. En este caso, dados los resultados del sistema se tiene que el umbral sería de \$356,994.63 (pesos) para Antioquia ${ }^{3}$. Se debe puntualizar que esta sería la línea de pobreza para una unidad

\footnotetext{
3 Según la Misión de Empalme para las Series de Pobreza (MESEP, 2011), en Colombia se considera pobre a quienes devengan menos de al mes (año 2011). Por otra parte, el Banco mundial (BM, 2012) considera que una persona es pobre si su ingreso es menor a US\$2 diarios (en paridad de poder de compra) o US\$60 al mes.
} 
de gasto y no se tiene en cuenta si hay escalas de equivalencia para captar diferencias entre los hogares.

Para el caso de los términos al cuadrado del sistema, si bien son pequeños, son la mayoría estadísticamente significativos y sus signos son relevantes desde el punto de vista económico. Según los resultados presentados en la cuadro 8 la participación del gasto en grupo de bienes como alimentos, vivienda, salud, y bebidas-servicios personales descienden sobre el gasto total a medida que aumenta el ingreso, mientras que bienes como educación, cultura y bienes durables ganan participación en el gasto total a medida que aumenta el ingreso. Además de los anteriores resultados, el sistema cuadrático estimado tiene altos coeficientes de ajuste.

Una vez realizada la estimación del sistema se logró establecer que el sistema presenta heteroscedasticidad en todas las ecuaciones del modelo. No obstante es necesario recordar, como anota Wooldridge (2002), que cuando existe éste problema los mínimos cuadrados ordinarios son aún insesgados y consistentes. Es decir, el problema de heteroscedasticidad es más que todo un problema de eficiencia en los estimadores, ya que su varianza deja de ser mínima y las pruebas de hipótesis sobre la significancia de los parámetros tendrán problemas ya que los intervalos de confianza serán mayores, lo que lleva posiblemente a aceptar parámetros significativos cuando no lo son.

Esta situación llevó a que se realizara la transformación de varianza de White que ajusta la matriz de varianzas-covarianzas y permite realizar pruebas de hipótesis robustas ante la presencia de heteroscedasticidad. Mediante esta transformación se encuentra que los parámetros estimados con mínimos cuadrados sí son significativos y las estimaciones están en el anexo A.

\subsection{Análisis comparativo con otros resultados}

Una vez estimado el sistema cuadrático para Antioquia, se realizan a continuación algunas comparaciones con los resultados obtenidos en otros trabajos.

El sistema cuadrático fue estimado en un primer momento para Colombia por Niño (2001) quien analiza los patrones de consumo de las cuatro principales ciudades del país. Buscaba con ello analizar posibles curvaturas en las curvas de Engel haciendo uso de la encuesta de gastos e ingresos 1994-1995 del DANE.

Niño (2001) estima el sistema cuadrático de gastos, y en lo que respecta a los consumos de subsistencia estimados en dicho trabajo, cuando la autora utiliza el sistema cuadrático de gastos encuentra para la ciudad de Medellín un gasto mínimo de subsistencia de \$62132 a pesos de 1995. En este caso, si se utiliza la variación 
del IPC solo de alimentos como proxy a una cifra de inflación ${ }^{4}$ promedio en estos años, la cual es de $10.10 \%$ según el DANE, el gasto mínimo de subsistencia sería a pesos de 2008 igual a $\$ 257,858$.8. Esta cifra es inferior en $\$ 99,135$ con respecto a la estimada en el presente trabajo.

Debido a la falta de trabajos que utilicen el sistema cuadrático para estimar consumos de subsistencias en Colombia, se hacen comparaciones con otros trabajos más recientes que han estimado otros sistemas para calcular líneas de pobreza.

El consumo de subsistencia para Colombia ha sido estimado también con el llamado sistema lineal de gastos. Es necesario recordar que dicho sistema es un caso particular del sistema cuadrático estimado acá, ya que ese sistema no capta las no-linealidades de las curvas ingreso consumo (Howe, 1974). La comparación es difícil realizarla con otro sistema de demanda ya que solo los sistemas de gastos han sido usados a gran escala para estimar los gastos de subsistencia. De hecho en los sistemas flexibles, tipo AIDS, Rotterdam, QAIDS, entre otros, es difícil argumentar desde la teoría que algún parámetro pueda interpretarse como un gasto de subsistencia. Por tanto, la comparación más idónea sería con las estimaciones que se han hecho del sistema lineal de gastos.

Para efectos de comparación, y haciendo énfasis en que el sistema lineal de gastos es un caso especial del sistema cuadrático, se referencian a continuación algunos resultados arrojados con la estimación del sistema lineal de gastos. Para Colombia algunas estimaciones de este sistema son las siguientes.

Esta el trabajo de Rivas (2000) quien estima el sistema lineal de gastos con datos de la encuesta de ingresos y gastos del DANE año 1994-1995 y, para el caso de Medellín, los resultados son:

Consumo de subsistencia en alimentos: $\gamma_{1}=\$ 30,074$

Consumo de subsistencia en todos los grupos de bienes: $\sum_{i=1}^{12} \gamma_{i}=\$ 90,784$

Para comparar los resultados con los encontrados en el presente trabajo, se podría utilizar la tasa de inflación promedio entre los años 1995-2008, la cual según el DANE sería de $10.10 \%$. En este caso, se tendría que el consumo de subsistencia en alimentos sería $\gamma_{1}=\$ 124,812$ y el consumo de subsistencia en los doce grupo de bienes $\sum_{i=1}^{12} \gamma_{i}=\$ 376,769$.

Se utiliza sólo la variación del IPC de alimentos, debido a que la variación del IPC total incorpora también la variación de otros grupos de gastos no tomados en cuenta en este documento. 
Para el caso de alimentos, se tiene entonces que el sistema cuadrático arroja entonces un resultado de casi el $\mathbf{5 1 \%}$ más alto en el consumo de subsistencia de alimentos. En el consumo de subsistencia total, los resultados encontrados con el sistema cuadrático son más bajos en \$19,774.

Se traen ahora los resultados de Cortés y Pérez (2010) quienes utilizan la encuesta de ingresos y gastos del DANE año 2006-2007. Para el caso de los alimentos, cuando los autores utilizan el sistema lineal de gastos, se encuentra que el consumo de subsistencia de alimentos para Colombia es $\gamma_{1}=\$ 366,610$ y el consumo de subsistencia en los diferentes grupos de bienes sería igual a $\sum \gamma_{i}=\$ 1,596,520$ . De nuevo en este caso, el gasto de subsistencia en alimentos como el gasto de subsistencia en el total de bienes es mucho más alto que los resultados encontrados en el presente trabajo con el sistema cuadrático.

Por último, se referencia el trabajo de Muñoz (2009). Para efectos de comparación se extraen solo los resultados estimados con el sistema lineal de gastos extendido simple donde se llega a que el gasto mínimo de subsistencia total es $\sum \gamma_{i}=\$ 287,499$ , en este caso para Bogotá. En este caso, con una inflación promedio de 7,44\% entre 2004-2008 con base en la variación del IPC de alimentos, se tendría que la línea de pobreza medida con el gasto de subsistencia sería \$411,591 a pesos del 2008 para la ciudad de Bogotá, un resultado \$54,596 más alto al encontrado en el presente trabajo para la región de Antioquia.

En síntesis, de los resultados encontrados acá se puede afirmar que van acorde con la literatura especializada en el tema para Colombia. Como se anotaba más arriba, las comparaciones anteriores se hicieron solo para efectos de contraste con los resultados encontrados con el sistema cuadrático. Solo sirven como punto de referencia ya que si se usa el sistema cuadrático, el consumo es una función no lineal del ingreso, lo cual significa que la participación del gasto puede variar para hogares con diferentes niveles de ingresos. Además, las estimaciones empíricas muestran a menudo que la función ingreso-consumo es no lineal.

Algunas estimaciones no paramétricas para la región de Antioquia, que soportan la no linealidad de las curvas de Engel para algunos bienes, fueron realizadas en el presente trabajo y se encuentran al final en el anexo C.

\section{ELASTICIDADES PRECIO PROPIO, CRUZADA Y GASTO DE LA DEMANDA}

Una vez estimado el consumo de subsistencia y caracterizada la composición y distribución del gasto en Antioquia, se plantea ahora un sistema de ecuaciones de demanda que permita estimar, no solo las elasticidades gasto, sino también las 
elasticidades precio propio y cruzada de la demanda para con estas analizar posibles patrones de consumo en la región.

El sistema de demanda a utilizar es un sistema de las llamadas formas flexibles que se diferencia por las deseables propiedades teóricas que traen en el cálculo de las elasticidades. Estos sistemas no presentan, por ejemplo, el llamado problema de identificación tan crónico en los sistemas de gastos lineales o cuadráticos. Además de ello, la inclusión de suficientes variables permite que las elasticidades no caigan entre rangos como sucede con los sistemas de gastos. Es por ello que en esta parte del trabajo, que intenta estimar elasticidades, se utiliza un sistema flexible.

Para realizar el cálculo de las elasticidades se utiliza el sistema casi ideal de demanda (AIDS) definido en forma estocástica como:

$$
w_{i}=\alpha_{i}+\sum_{j=1}^{L} \gamma_{i j} \ln p_{j}+\beta_{i} \ln \left(\frac{m}{P}\right)+e_{i}
$$

Donde:

$w_{i}$ es la participación en el gasto del bien i y $m$ es la renta. $a_{i}, g_{i j}$ y $\beta_{i}$ son constantes. Se supone que el término cumple con $\mathrm{E}(e)=0$ pero $V\left(e_{i}\right)=\tilde{U}$ es una matriz de varianzas-covarianzas particular ya que se estimará el modelo por el método ITSUR.

Por otra parte, P es el índice de precios del sistema y en este trabajó se optó por hacer uso del índice de Laspeyres

$$
\ln P=\sum_{k=1}^{L} w_{k}{ }^{0} \ln \left(p_{k}\right)
$$

con $w_{k}^{0}$ la participación del gasto $k$ en el período base, la cual se aproxima mediante el promedio de la participación de dicho gasto y $p_{k}$ el precio del bien $k$. Este índice de precios ha mostrado que tiene potencial para reducir el problema de la simultaneidad (ver Moschini, 1995).

Es necesario contar con un índice de precios para la estimación del sistema AIDS, ya que si se supone que los precios son constantes, el sistema sería idéntico al Working-Leser y solo se podrían estimar las elasticidades gasto. Para realizar el cálculo del índice de precios se siguió la metodología propuesta por Lewbel (1989) y Perali, Atella y Menon (2004) la cual fue implementada para Colombia por Ballesteros (2011). Este método parte del hecho de que las encuestas de hogares, tal como la encuesta de calidad de vida utilizada en esta investigación, solo reportan la información sobre el gasto de los hogares y no traen la información sobre las cantidades compradas, excluyéndose así la posibilidad de derivar los valores específicos de las unidades compradas. 
El método de Perali, Atella y Menon (2004) está basado en el desarrollo teórico de Lewbel (1986) de equivalencias de escala que depende de los gastos de los hogares y construye con ellas el llamado pseudo índice de precios de valores unitarios para un grupo de gastos. Mediante este método se llega que el pseudo índice de precios de valores unitarios $\widehat{P}_{i}$ para un grupo de gastos es:

$$
\widehat{P}_{i}=\frac{\prod_{j=1}^{n} w_{i j}{ }^{-w_{i j}}}{\prod_{j=1}^{n} w_{i j}{ }^{R-w_{i j}{ }^{R}}}
$$

Donde $w_{i j}$ es la participación en el gasto $i$ del bien $j$ y $w_{i j}{ }^{R}$ es la participación en el gasto $i$ del bien $j$ para el hogar de referencia o promedio. Se supone que este pseudo índice de precios de valores unitarios es para una región, Antioquia. Solo hay un paso para pasar de este pseudo índice de precios de valores unitarios a los pseudo valores unitarios y consiste en multiplicar el índice de arriba por el gasto promedio en cada uno de los grupos de la región considerada.

$$
\widehat{P_{L}}=\widehat{P}_{i} *\left(y_{i}\right)
$$

Donde $y_{i}$ es el gasto promedio. $\widehat{P_{L}}$ es entonces los pseudo valores unitarios o precios implícitos en los gastos de los hogares. Esta es entonces una forma de calcular los precios de los gastos de los hogares a partir de los datos disponibles y, ya que las participaciones del gasto en cada uno de los grupos de bienes que se definieron en secciones anteriores sí se conocen, es posible proceder así con el cálculo de los precios implícitos.

\subsection{Cálculo de precios implícitos en la ECV}

El método para calcular los precios implícitos presentado antes se hace necesario para construir una serie de precios para cada hogar con base en sus gastos que permita realizar cálculos de elasticidades confiables.

En este trabajo se consideró que para una encuesta tipo corte transversal como la utilizada acá, una buena aproximación a una serie de precios es el método de precios implícitos, aunque es necesario aclarar que el método tiene falencias. Por ejemplo, para calcular los precios se requiere que las observaciones de los gastos de los hogares en los distintos subgrupos sean distintas de cero y existan varios subgrupos de gasto por cada grupo de bienes (Ballesteros, 2011).

En el caso del presente trabajo, el grupo de alimentos está conformado de seis subgrupos: harinas y cereales, leche y huevos, carnes, frutas, hortalizas y leguminosas, plátanos y tubérculos, alimentos varios. Igual como se definió para el caso 
de alimentos se procedió a estimar cada pseudo índice para el grupo de vivienda, salud, transporte y comunicaciones, educación, cultura y recreación, bebidas, tabaco y servicios personales, y bienes durables y vestidos. Es decir, siete grupos de pseudo índices definidos de manera ad hoc intentado tener la mayor cantidad de precios positivos. Se debe reconocer así que la agregación que se hace es alta.

Con base en esto se procedió a formar los siguientes subgrupos.

Cuadro 9. Clasificación de gastos y subgrupos

\begin{tabular}{|c|c|c|c|}
\hline No. & Grupo & No. & Subgrupo \\
\hline \multirow{5}{*}{1} & \multirow{5}{*}{ Alimentos } & 1.1 & Harinas y cereales \\
\hline & & 1.2 & Leche y huevos \\
\hline & & 1.3 & Carnes \\
\hline & & 1.4 & Frutas, hortalizas, leguminosas, plátanos y tubérculos \\
\hline & & 1.5 & Alimentos varios y enlatados \\
\hline \multirow{2}{*}{2} & \multirow[t]{2}{*}{ Vivienda } & 2.1 & Arrendamientos y predial \\
\hline & & 2.2 & Servicios públicos \\
\hline \multirow{2}{*}{3} & \multirow[t]{2}{*}{ Salud } & 3.1 & Hospitalización y servicios de hospitalización \\
\hline & & 3.2 & Bienes y servicios de saludo \\
\hline \multirow{2}{*}{4} & \multirow{2}{*}{ Transporte } & 4.1 & Transporte público \\
\hline & & 4.2 & Uso vehículo particular \\
\hline \multirow{2}{*}{5} & \multirow{2}{*}{$\begin{array}{l}\text { Educación, cultura } \\
\text { y recreación }\end{array}$} & 5.1 & Artículos de recreación y culturales e instrucción \\
\hline & & 5.2 & Artículos para la educación \\
\hline \multirow{2}{*}{6} & \multirow{2}{*}{$\begin{array}{l}\text { Bebidas, tabaco y } \\
\text { servicios p. }\end{array}$} & 6.1 & Artículos de servicio personal \\
\hline & & 6.2 & Bebidas alcohólicas y tabaco \\
\hline \multirow{2}{*}{7} & \multirow{2}{*}{$\begin{array}{l}\text { Bienes durables y } \\
\text { vestido }\end{array}$} & 7.1 & Ropa y artículos conexos. \\
\hline & & 7.2 & Bienes durables, Seguros y tarjeta de crédito. \\
\hline
\end{tabular}

Fuente: elaboración propia.

Una vez definidos los grupos y subgrupos se procedió a estimar los pseudo valores unitarios implícitos en el gasto de los hogares. Se presentaron varios problemas, y el principal es la existencia de una considerable cantidad de observaciones ceros en muchos de los subgrupos, problema que es común a las tres encuestas de calidad de vida que hace el DANE. 
Para cada grupo de gastos se pudieron construir los siguientes pseudo precios.

Cuadro 10. Calculo de pseudo precios

\begin{tabular}{|c|l|c|c|}
\hline No. & \multicolumn{1}{|c|}{ Grupo } & Total pseudo precios calculados & Media \\
\hline 1 & Alimentos & 1059 & $223,167.08$ \\
\hline 2 & Vivienda & 1693 & $272,968.18$ \\
\hline 3 & Salud & 664 & $30,830.6$ \\
\hline 4 & Transporte & 625 & $40,371.11$ \\
\hline 5 & Educación, cultura y recreación & 603 & $27,221.12$ \\
\hline 6 & Bebidas, tabaco y servicios p. & 416 & $14,770.93$ \\
\hline 7 & Bienes durables y vestido & 482 & $36,238.19$ \\
\hline
\end{tabular}

Fuente: elaboración propia con información de la ECV, DANE (2008).

Según se observa, en los grupos de gastos 6 y 7 la construcción de precios es bastante limitada ya que se obtiene menos del $40 \%$ de precios para las familias. Sin embargo, serán las estimaciones econométricas las que decidan la significancia de la serie de precios construida para cada grupo de gastos.

\subsection{Estimación del sistema AIDS}

Una vez construida la serie de precios y el índice de precios global se estima el sistema casi ideal de demanda mediante el método de regresiones iterativas aparentemente no relacionadas, ITSUR, implementado en SAS con la ayuda de algunos ejemplos del manual de programa SAS (2002).

Con este procedimiento se estiman el conjunto de ecuaciones del modelo y no se impusieron las propiedades de la teoría del consumidor mencionadas antes como restricciones ya que en el método se deja una ecuación libre que es estimada por las propiedades necesarias para que el modelo cumpla las restricciones teóricas. Es decir, del total de los siete grupos de bienes se deja una ecuación libre y los parámetros de esta son estimados mediante las propiedades del modelo. De este modo se puede evitar que las perturbaciones en la estimación de una ecuación estén correlacionadas con las de otra.

El método ITSUR supone que la matriz de varianza-covarianzas de los errores, $V\left(e_{i}\right)=\tilde{U}$, tiene la característica de no ser escalar ya que se admite correlación contemporánea. Esto es importante ya que en caso de utilizar mínimos cuadrados ordinarios no se estaría haciendo uso de la información contenida en la covarianza contemporánea de las diferentes ecuaciones. Los resultados se muestran en la cuadro 11 . 
El consumo y la línea de pobreza en Antioquia: aproximaciones mediante la teoría del consumidor

Cuadro 11. Estimación del SCG para Antioquia, 2008

\begin{tabular}{|c|c|c|c|c|c|c|c|c|c|c|c|c|}
\hline MODEL & DEPVAR & Intercept & Inpal & Inpviv & Inpsal & Inptrans & Inpculyedu & & Inpserybeb & & npduryves & mreal \\
\hline ALIM & w1 & $* \star *, 52707$ & $* * * \quad 0,00543$ & $* * *-0,00452$ & ${ }^{* \star *}-0,00472$ & ${ }^{* \star *}-0,00315$ & $* * * \quad-0,00266$ & $\dagger$ & $-0,00114$ & *** & $-0,00501$ & ${ }^{* * *}-0,00714$ \\
\hline VIV & w2 & *** $\quad 0,35798$ & ${ }^{* \star *}-0,00656$ & ${ }^{* \star *} \quad 0,00568$ & $* \star * \star 0,00195$ & ${ }^{* * *}-0,00414$ & $\dagger-0,00109$ & *** & $-0,00367$ & *** & $-0,00480$ & ${ }^{* \star *}-0,00497$ \\
\hline SALUD & w3 & $\dagger-0,02393$ & $* \star * * 00182$ & $+0,00077$ & *** $\quad 0,00893$ & $\dagger-0,00008$ & * $-0,00075$ & ** & $-0,00085$ & $\dagger$ & $-0,00073$ & ${ }^{* * *} \quad 0,00570$ \\
\hline TRANS & w4 & *** $\quad 0,04794$ & $\dagger 0,00026$ & ${ }^{* \star *}-0,00197$ & $\dagger 0,00001$ & 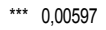 & $\dagger-0,00027$ & $\dagger$ & 0,00015 & *** & 0,00128 & ${ }^{* * *} \quad 0,00284$ \\
\hline EDUCULT & w5 & ${ }^{* * *} \quad 0,03445$ & $\dagger-0,00025$ & $\dagger-0,00031$ & $\dagger-0,00003$ & $\dagger 0,00030$ & $* * * \quad 0,00420$ & $\dagger$ & $-0,00041$ & $\dagger$ & $-0,00034$ & $\dagger 0,00052$ \\
\hline DURYVES & w7 & ${ }^{* * \star}-0,04277$ & ${ }^{* * *} \quad 0,00128$ & ${ }^{* * *} \quad 0,00226$ & ${ }^{*}-0,00072$ & ${ }^{* * *} \quad 0,00145$ & * 0,00075 & *** & $-0,00109$ & *** & 0,00940 & $* * * \quad 0,00522$ \\
\hline BEBYSER $\ddagger$ & w6 & 0,09927 & $-0,00114$ & $-0,00367$ & $-0,00085$ & 0,00015 & $-0,00041$ & & 0,00701 & & $-0,00109$ & $-0,00216$ \\
\hline
\end{tabular}

Notas: ***Significativo al 1\%,**Significativo al 5\%, * Significativo al 10\%, 4 No Significativo. $\ddagger$ El modelo de BEBYSER fue estimado por residuo.

Fuente: Elaboración propia con información de la ECV, DANE (2008).

En total se estimaron 6 ecuaciones y 54 parámetros a partir de las observaciones de los hogares de la encuesta de calidad de vida 2008. En su mayoría los coeficientes son significativos al 1\% y 5\% según la prueba de significancia individual. En el sistema estimado, hay un total de 17 parámetros no significativos (30\% del total), y la ecuación de transporte es la ecuación con mayor número de parámetros no significativos. No obstante, todos los parámetros del precio propio y la renta real de cada grupo son significativos y, por tanto, el ejercicio de construcción de los precios no es ocioso.

La ecuación libre elegida fue la del grupo de bebidas por ser el grupo donde menos precios implícitos se pudieron construir. En concreto los parámetros de la ecuación libre fueron estimados a partir de las restricciones que tiene el sistema para que aquel cumpla la ley de Walras y la homogeneidad de grado cero en precios y renta.

Para este sistema las elasticidades gasto, precio propio y elasticidad cruzada de la demanda son:

$$
\begin{aligned}
& \varepsilon_{i}=1+\frac{\beta_{i}}{w_{i}} \\
& \varepsilon_{i i}=-1+\frac{\gamma_{i i}}{w_{i}}-\beta_{i} \\
& \varepsilon_{i j}=\frac{\gamma_{i j}}{w_{i}}-\beta_{i} \frac{w_{j}}{w_{i}}
\end{aligned}
$$


Donde cada $w_{i}, \forall i=1,2, \ldots, L$ se aproxima en el punto medio. Las elasticidades se presentan a continuación en el cuadro 12.

Según los resultados del cuadro 12, todas las elasticidades gasto son positivas (bienes normales). Elasticidades menores a uno implican bienes necesarios y, por tanto, los alimentos, los servicios de vivienda, bebidas y servicios personales son bienes necesarios. Grupos de gastos como salud, transporte, educación y cultura y bienes durables, al tener elasticidad gasto mayor a uno, se comportan como bienes de lujo.

Estos resultados son similares con los encontrados por Ballesteros (2011). En este trabajo se encuentra que grupos de bienes como alimentos y vivienda son bienes necesarios y grupos de bienes como salud, transporte, educación, cultura y recreación y servicios personales son de lujo.

Cuadro 12. Elasticidades gasto y precio para Antioquia, 2008

\begin{tabular}{|c|c|c|c|c|c|c|c|c|}
\hline & G.TOTAL & P ALIM & P VIV & P SALUD & P TRANS & P EDUCULT & P BEBYSER & P DURYVES \\
\hline ALIM & 0,976856 * & $-0,97527$ * & $-0,0087$ * & $-0,01343$ * & $-0,00792$ * & $-0,00718769$ * & $-0,002603521 \dagger$ & $-0,012894903$ * \\
\hline VIV & 0,980725 * & $-0,0195$ * & $-0,9730$ * & $-0,00599$ * & $-0,01412$ * & $-0,003044451 \dagger$ & $-0,013321062$ * & $-0,015824122$ * \\
\hline SALUD & 1,070256 * & 0,000731 * & $-0,0086 \dagger$ & $-0,89572$ & $-0,00799 \dagger$ & $-0,013569995$ * & $-0,013776039$ * & $-0,019145896 \dagger$ \\
\hline TRANS & 1,0286 * & $-0,00624 \dagger$ & $-0,0272$ * & $-0,00221 \dagger$ & $-0,94275$ * & $-0,004463353 \dagger$ & $0,000168227 \dagger$ & 0,008778127 * \\
\hline EDUCULT & 1,008357 * & $-0,00655 \dagger$ & $-0,0072 \dagger$ & $-0,00112 \dagger$ & $0,00405 \dagger$ & $-0,932588228$ & $-0,007036401 \dagger$ & $-0,006762102 \dagger$ \\
\hline BEBYSER & 0,954096 & $-0,01005$ & $-0,0662$ & $-0,01434$ & 0,007752 & $-0,005884197$ & $-1,020901603$ & $-0,016448123$ \\
\hline DURYVES & 1,036201 * & $-0,00228$ * & 0,0064 * & * $-0,00793$ * & 0,006504 * & 0,002962019 * & $-0,009236176$ * & $-0,939937233$ * \\
\hline
\end{tabular}

Notas: *Significativo al 5\%, † No Significativo.

Fuente: elaboración propia con información de la ECV, DANE (2008).

Similares resultados encuentran Cortés y Pérez (2010) con la encuesta de gastos e ingresos 2006-2007. Con respecto a las elasticidades precio propios, en el cuadro 12 se observa que grupos como alimentos, vivienda, salud, transporte y durables son bienes inelásticos. Por otro lado, el grupo de gastos de bebidas y servicios personales se comportan como bienes elásticos, es decir, es el grupo de gastos con mayor sensibilidad ante los cambios en los precios propios. Le siguen los alimentos, vivienda, transporte, bienes durables, educación y cultura y, por último, salud.

En cuanto a las elasticidades precio, si se compara los resultados encontrados con los de otros trabajos, se encuentra que estos difieren con los de Ballesteros (2011) en gran proporción ya que en dicho trabajo se encuentra que todos los grupos de gasto tienen elasticidad precio propio mayor a uno. Por otra parte, Cortés y Pérez 
(2010) encuentran que los grupos de bienes alimentos, vivienda y salud tienen elasticidad precio propio mayor a uno y grupos como educación, transporte, vestidos y otros bienes son bienes inelásticos.

En cuanto a la homoscedasticidad de los residuales de cada una de las ecuaciones del sistema AIDS, es necesario aclarar que de nuevo se encuentra que hay problemas de heteroscedasticidad. Para ver las posibles consecuencias de esta sobre la significancia de los parámetros, se estimó el sistema con la corrección de White (anexo B) y se encontró que los parámetros significativos se mantienen.

\section{COMENTARIOS FINALES}

Mediante una aplicación de la teoría del consumidor, se ha estimado el sistema cuadrático de gastos con el fin de encontrar una aproximación para el consumo de subsistencia en la región Antioquia. Se encuentra que el consumo de subsistencia en los 7 grupos de bienes es igual a \$356,994.63.

Los alimentos son el grupo de bienes que más pesa en la zona rural con casi un 50\% mientras que en la zona urbana, si bien también son el grupo que más pesa en el gasto corriente, solo lo hacen con el $29 \%$. Se verifica, además, la Ley de Engel que afirma que a mayor proporción del gasto en alimentos sobre el gasto total, menor calidad de vida. Para el caso de la zona rural, el gran peso que tienen los alimentos trae como resultado que algunos bienes casi que no participen en el gasto y la calidad de vida sea inferior con respecto a la zona urbana.

Existe también evidencia de que ciertos grupos de bienes como alimentos, vivienda, salud, bebidas y servicios personales presenten curvas de Engel no lineales y que estas curvas, después de cierto nivel de ingreso, tengan pendientes negativas (se vuelven bienes inferiores). Por otra parte, grupos de bienes como educación y cultura, y bienes durables es probable presenten curvas de Engel con pendiente positiva después de cierto umbral de ingreso (ver anexo C).

Para investigaciones futuras queda abierto el campo para incluir variables demográficas al sistema cuadrático de gastos y ver las implicaciones que tiene la llegada de nuevos miembros al hogar, además de tratar de verificar cómo cambia el consumo de subsistencia en la región.

Se debe tener en cuenta que el estudio del consumo en Antioquia hecho en el presente trabajo para el año 2008, es acotado en cuanto es solo un primer paso para un marco de referencia más amplio. Es evidente que si se desea estudiar los cambios en el bienestar y la evolución de la calidad de vida en Antioquia, se debe extender el estudio hecho acá con las encuestas de calidad de vida 2003 y las encuestas de gastos anteriores, buscándose así un punto de vista con mayores horizontes. 


\section{BIBLIOGRAFÍA}

Altimir, Oscar (1979). Dimensión de la pobreza en América Latina. En: Serie Cuadernos de la Cepal, No. 27, 99p.

Ballesteros, Andrés (2011). Estimación de precios implícitos a partir de la información de gasto contenida en las encuestas de calidad de vida del Dane, Trabajo final para optar al título de Magíster en Ciencias Económicas, Universidad Nacional de Colombia, 67p.

Banco Mundial (2012). Estadísticas sobre pobreza. Disponibles en http://datos.bancomundial. org/tema/pobreza. Consultado en Octubre de 2011.

Cortés, D. y Pérez, Jorge (2010). El consumo de los hogares colombianos 2006-2007: estimación de sistemas de demanda. En: Documentos de trabajo, No. 86, Universidad del Rosario, 28p.

DANE -Departamento Administrativo Nacional de Estadística- (2008). Encuesta de calidad de vida.

Deaton, Angus y Muellbauer, John (1983). Economics and Consumer Behavior, Cambridge University Press, Cambridge, 450p.

Deaton, Angus y Muellbauer, John (1980). An Almost Ideal Demand System. En: American Economic Review, Vol.70, No. 3, p. 312-326.

Feres, Juan C. y Mancero, Xavier (2001). Enfoques para la medición de la pobreza. Breve revisión de la literatura. En: Serie estudios estadísticos y prospectivos, No. 04, CEPAL, 48p.

Howe, Howard; Pollak, Robert y Wales, Terence (1979). Theory and time series estimation of the quadratic expenditure system. En: Econometrica, Vol.47, No. 5, p. 1231-1247.

Howe, H. (1974). Estimation of the Linear and Quadratic Expenditure System: A Cross-Section Case for. Colombia, Unpublished Ph.D. dissertation, University of Pennsylvania, 106p.

Lewbel, Arthur (1989). Identification and estimation of equivalence scales under weak separability. En: The Review of Economic Studies. Vol 56, No. 2, p. 311-316.

Lluch, C. y Williams, R. (1975). Cross Country Demand and Savings Patterns: An Application of the Extended Linear Expenditure System. En: The Review of Economics and Statistics, Vol. 57, No. 3, p. 320-328.

Mas-Colell, Andreu; Whinston, Michael. y Green, Jerry (1995). Microeconomic Theory. Oxford University Press, Oxford, 986p.

MESEP -Misión para el Empalme de las Series de Empleo, Pobreza y Desigualdad. (2011). Nueva metodología para la medición de la pobreza monetaria y cifras de pobreza extrema, pobreza y desigualdad 2002-2010. DANE-DNP, http://www.dnp.gov.co/LinkClick.aspx?fileticket=DX InD1TENeU\%3DEtabid=337 (Consultado en Octubre de 2011).

Moschini, Giancarlo (1995). Units of Measurement and the Stone Index in Demand System Estimation. En: American Journal of Agriculture Economics, Vol. 77, No. 1, p. 63-68.

Muñoz, Ayala Jorge (2009). Estimación de coeficientes de Orshansky a partir de un sistema completo de ecuaciones de demanda: una nueva metodología para la elaboración de umbrales de pobreza. En: Cuadernos de economía, No. 50, p. 191-221. 
Muñoz, Manuel; Ramírez Manuel y Zambrano, Andrés (2005). Comparación del gasto de los hogares colombianos entre 1997 y 2003, según resultados de las encuestas de calidad de vida: magnitud, composición y distribución. En: Borradores de investigación, No. 01, Universidad del Rosario, 46p.

Muñoz, Manuel (1990). La pobreza medida a través de ingresos y gastos: Un replanteamiento. En: Boletín de Estadística. No. 450, 40p.

Nicholson, J. L. (1949). Variations in Wonking-Classs family expenditure. En: Journal of the Royal Statistical Society, Vol. 112, p. 359-391.

Niño, Claudia (2001). Estimación de sistemas completos de ecuaciones de demanda, Tesis para optar el título de Magíster en Economía, Universidad Nacional de Colombia, 78p.

Perali, Federico. Vicenio Atella y Mariana Menon (2004). Estimation of unit values in cross sections without quantity information and implications for demand and welfare analysis. En: Dagum, Camilo y Ferrari, Guido (eds). Household behavior, equivalence scales, welfare and poverty, Springer-Verlag Company, New York, p. 195-220.

Rivas M., Guillermo (2000). Patrones de demanda de los Hogares en las Cuatro Principales Ciudades de Colombia: Bogotá, Cali, Medellín y Barranquilla, Tesis para optar el título de Magíster en Economía, Universidad Nacional de Colombia, 94p.

Samuelson, Paul (1947). Some Implications of Linearity. En: Review of Economic Studies, No. 15, p. 88-90.

SAS System (2002). Estimating an Almost Ideal Demand System Model. SAS/ETS. Examples. http:// support.sas.com/rnd/app/examples/ets/aids/index.htm, (Consultado el 19 de Agosto de 2011).

Sen, Amartya (2000). Desarrollo y Liberad. Planeta Ediciones, España, 440p.

Sen, Amartya (1992). Sobre conceptos y medidas de pobreza. En: Revista Comercio Exterior, Vol. 42, No. 4, p. 310-322.

Stone, Richard (1954). Linear Expenditure Systems and Demand Analysis: An Application to the Pattern of British Demand. En: Economic Journal, Vol. 64, p. 511-527.

Wooldridge, Jeffrey (2002). Econometric Analysis of Cross Section and Panel Data, The MIT Press, Cambridge, $752 \mathrm{p}$. 
Anexo A. Estimación del Sistema cuadrático de gastos con corrección de White para Antioquia, 2008

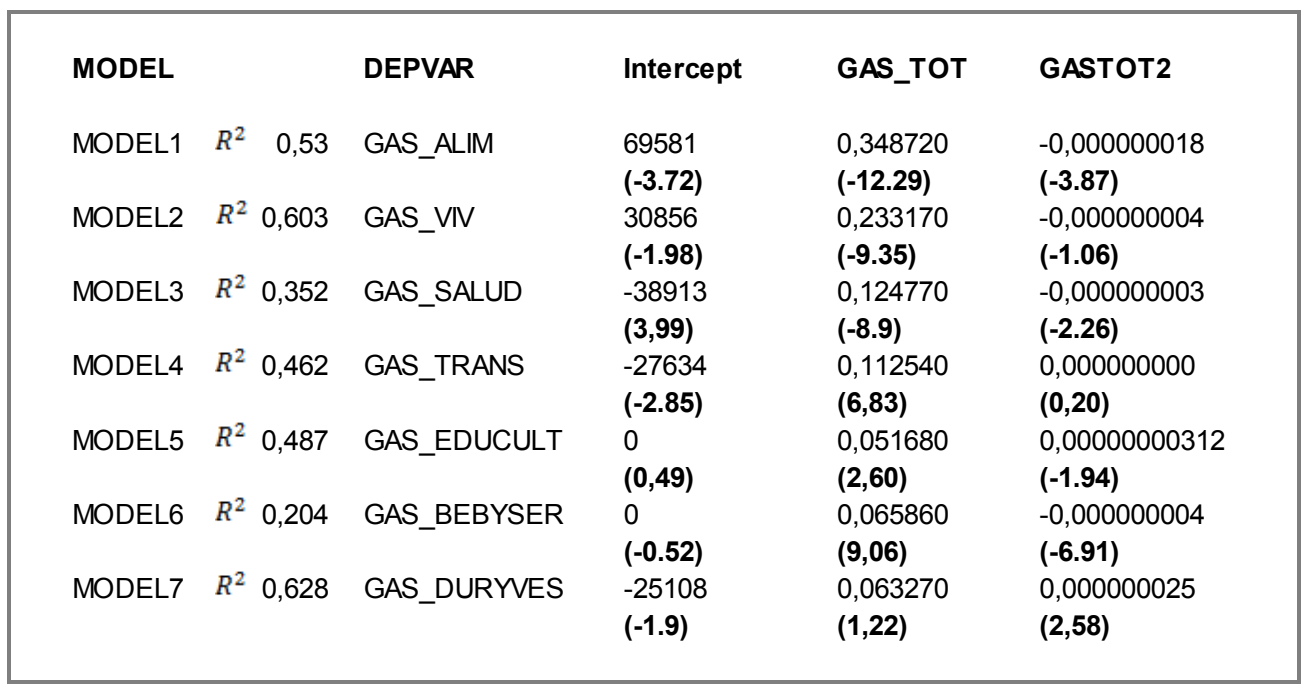

Notas: Entre paréntesis están los valores t de los parámetros. Un valor t mayor a $|1.9|$ indica que el parámetro es significativo.

Fuente: Elaboración propia con información de la ECV, DANE (2008).

Anexo B. Estimación del Sistema AIDS para Antioquia con ajuste White, 2008

\begin{tabular}{|c|c|c|c|c|c|c|c|c|c|c|c|c|c|c|}
\hline MODEL & DEPVAR & Intercept & Inpal & Inpviv & Inpsal & & Inptrans & & Inpculyedu & & npserybeb & & Inpduryves & mreal \\
\hline ALIM & w1 & $\begin{array}{r}* * 52707 \\
\\
(14,38)\end{array}$ & $\begin{array}{r}0,00543 \\
(\mathbf{4}, \mathbf{7 1})\end{array}$ & $\begin{array}{r}* \star * \\
-0,00452 \\
(-2.6)\end{array}$ & $\begin{array}{r}* * * \\
-0,00472 \\
(-5.7)\end{array}$ & $* * *$ & $\begin{array}{r}-0,00315 \\
(-3.9)\end{array}$ & $\star \star \star *$ & $\begin{array}{r}-0,00266 \\
(3,05)\end{array}$ & $\dagger$ & $\begin{array}{r}-0,00114 \\
(\mathbf{1}, \mathbf{2 0})\end{array}$ & *** & $\begin{array}{r}-0,00501 \\
(-5.3)\end{array}$ & $\begin{array}{r}* * *-0,00714 \\
(-3.0)\end{array}$ \\
\hline VIV & w2 & $\begin{array}{rr}* \star * & 0,35798 \\
& (12,11)\end{array}$ & $\begin{array}{r}* * *-0,00656 \\
(-7.0)\end{array}$ & $\begin{array}{r}\text { *** } 0,00568 \\
\quad(\mathbf{4}, \mathbf{2 0})\end{array}$ & $\begin{array}{r}* * *-0,00195 \\
(-\mathbf{3 . 0})\end{array}$ & $\star \star * *$ & $\begin{array}{r}-0,00414 \\
(-5.8)\end{array}$ & $\dagger$ & $\begin{array}{r}-0,00109 \\
(-1.5)\end{array}$ & $* * *$ & $\begin{array}{r}-0,00367 \\
(-4.7)\end{array}$ & $\star \star * *$ & $\begin{array}{r}-0,00480 \\
(-6.2)\end{array}$ & $\begin{array}{r}* * \star-0,00497 \\
(-2.1)\end{array}$ \\
\hline SALUD & w3 & $\begin{array}{r}\dagger-0,02393 \\
(-1.23)\end{array}$ & $\begin{array}{r}\text { *** } 0,00182 \\
(\mathbf{3}, 15)\end{array}$ & $\begin{array}{r}0,00077 \\
(\mathbf{0 , 9 0 )}\end{array}$ & $\begin{array}{r}* * * \quad 00893 \\
\quad(17,89)\end{array}$ & $\dagger$ & $\begin{array}{r}-0,00008 \\
(-\mathbf{0 . 2})\end{array}$ & * & $\begin{array}{r}-0,00075 \\
(1,80)\end{array}$ & $* *$ & $\begin{array}{r}-0,00085 \\
(-1.93)\end{array}$ & $\dagger$ & $\begin{array}{r}-0,00073 \\
(-1.62)\end{array}$ & $\begin{array}{r}* * * 00570 \\
(\mathbf{4}, \mathbf{2 1})\end{array}$ \\
\hline TRANS & w4 & $\begin{array}{r}* * 04794 \\
(2,72)\end{array}$ & $\begin{array}{r}+0,00026 \\
(\mathbf{0 , 5 2})\end{array}$ & 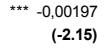 & $\begin{array}{r}0,00001 \\
(\mathbf{0 , 0 3})\end{array}$ & *** & $\begin{array}{r}0,00597 \\
(15,37)\end{array}$ & $\dagger$ & $\begin{array}{r}-0,00027 \\
(-0.71)\end{array}$ & $\dagger$ & $\begin{array}{r}0,00015 \\
(\mathbf{0}, \mathbf{3 8})\end{array}$ & $* \star *$ & $\begin{array}{r}0,00128 \\
(3,07)\end{array}$ & $\begin{array}{r}* * 00284 \\
(2,80)\end{array}$ \\
\hline EDUCULT & w5 & $\begin{array}{r}\text { *** } 0,03445 \\
\quad(2,66)\end{array}$ & $\begin{array}{r}\dagger-0,00025 \\
(-\mathbf{0 . 6 9 )}\end{array}$ & $\begin{array}{r}\dagger-0,00031 \\
(-0.54)\end{array}$ & $\begin{array}{c}\dagger-0,00003 \\
(-\mathbf{0 . 1 1})\end{array}$ & $\dagger$ & $\begin{array}{r}0,00030 \\
(\mathbf{1}, \mathbf{2 7})\end{array}$ & $* * *$ & $\begin{array}{r}0,00420 \\
(17,01)\end{array}$ & $\dagger$ & $\begin{array}{r}-0,00041 \\
(-1.61)\end{array}$ & $\dagger$ & $\begin{array}{r}-0,00034 \\
(-1.4)\end{array}$ & $\begin{array}{r}+\quad, 00052 \\
\mathbf{( 0 , 7 1 )}\end{array}$ \\
\hline DURYVES & w7 & $\begin{array}{r}* * *-0,04277 \\
(-2.2)\end{array}$ & $\begin{array}{r}* * 00128 \\
(2,00)\end{array}$ & $\begin{array}{r}\text { *** } \quad 00226 \\
\quad(3,94)\end{array}$ & $\begin{array}{c}{ }^{*}-0,00072 \\
(-1.84)\end{array}$ & $\star \star \star *$ & $\begin{array}{r}0,00145 \\
(\mathbf{3}, \mathbf{5 6})\end{array}$ & * & $\begin{array}{r}0,00075 \\
(\mathbf{1}, \mathbf{8 2})\end{array}$ & 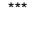 & $\begin{array}{r}-0,00109 \\
(-2.55)\end{array}$ & 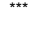 & $\begin{array}{r}0,00940 \\
(15,90)\end{array}$ & $\begin{array}{r}0,00522 \\
(3,37)\end{array}$ \\
\hline BEBYSER ‡ & w6 & 31,96927 & $-0,00114$ & $-0,00367$ & $-0,00085$ & & 0,00015 & & $-0,00041$ & & 0,00701 & & $-0,00109$ & 7,71784 \\
\hline
\end{tabular}

Notas: ***Significativo al 1\%, **Significativo al 5\%, *Significativo al 10\%, † No Significativo.

¥ El modelo de BEBYSER fue estimado por residuo.

Entre paréntesis están los valores t de los parámetros. Un valor t mayor a $|1.9|$ indica que el parámetro es significativo.

Fuente: Elaboración propia con información de la ECV, DANE (2008). 
Anexo C. Curvas de Engel para algunos grupos de bienes.

Los métodos no paramétricos no suponen una distribución específica de las variables y pueden estimar funciones no lineales. Se presenta a continuación algunas estimaciones gráficas de algunas curvas de Engel para ciertos grupos de bienes. El método usado fue el de Lowess y se utilizó el programa Stata.

Gráfico 1. Curva de Engel para alimentos para Antioquia

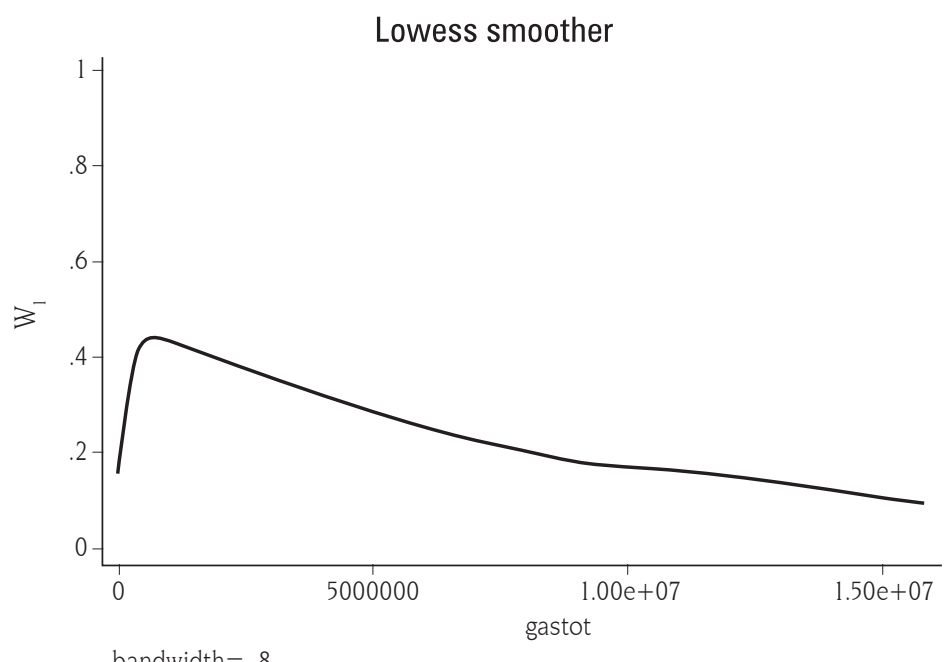

Fuente: Elaboración propia con información de la ECV, DANE (2008).

Según el gráfico 1, el gasto en alimentos crece, como proporción del gasto total, hasta un nivel de gasto de $\$ 1,200,000$ y llegar a ocupar el $w_{1}=45 \%$ del gasto. Después de este nivel de gasto, la proporción empieza a descender y en niveles altos de gasto solo ocupa un $10 \%$. 
Gráfico 2. Curva de Engel para salud para Antioquia

\section{Lowess smoother}

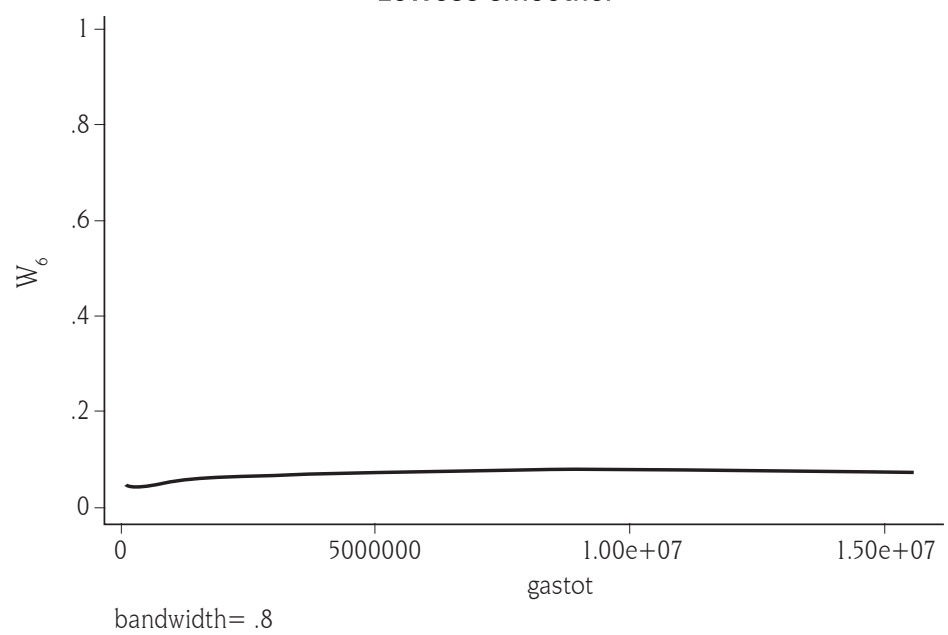

Fuente: Elaboración propia con información de la ECV, DANE (2008).

Según el gráfico 2, el gasto en salud crece a medida que el gasto total lo hace. Y se estabiliza como proporción del gasto total en un $w_{6}=0.8 \%$ después de que el gasto supera los $\$ 5,000,000$.

Gráfico 3. Curva de Engel para educación para Antioquia

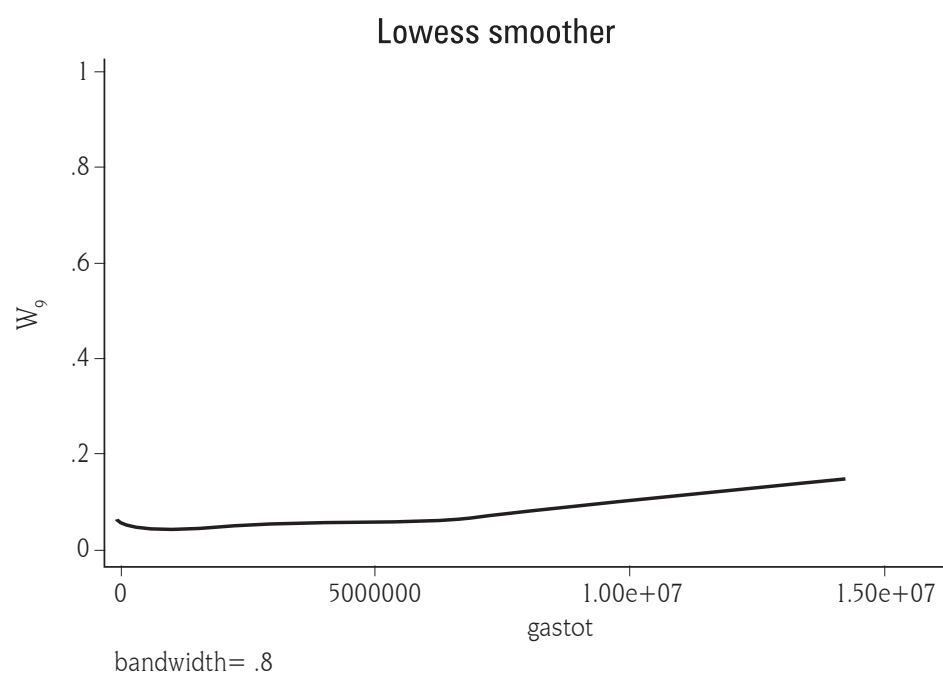

Fuente: Elaboración propia con información de la ECV, DANE (2008).

Según el gráfico 3, el gasto en educación se mantiene constante como proporción del gasto total en un nivel aproximado de $w_{9}=5 \%$ hasta cuando el gasto total 
llega, más o menos, a \$1,000,000. Después de este, la participación del gasto en educación como proporción del gasto siempre crece.

Gráfico 4. Curva de Engel para bienes durables para Antioquia

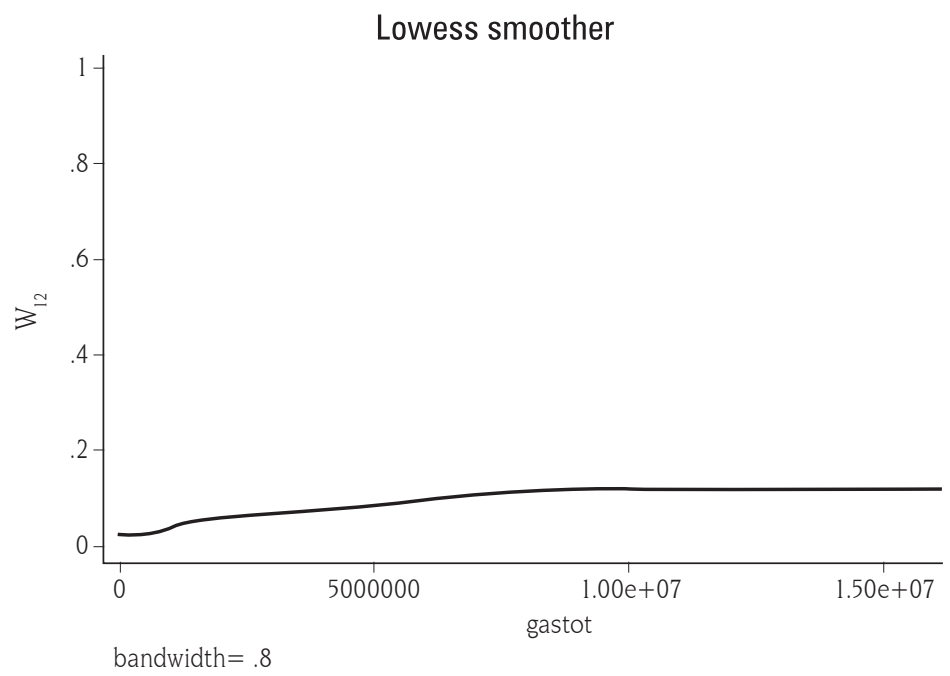

Fuente: Elaboración propia con información de la ECV, DANE (2008).

Por último, se presenta la curva de Engel para el gasto en bienes durables. Según el gráfico 4, la proporción del gasto en bienes durables siempre será crecientes hasta cuando el gasto llega más o menos a \$10,000,000 y la proporción a este nivel es $w_{12}=10 \%$. 